\title{
An Investigation of Silver Nanoparticles Formation under Presence of Graphene Quantum Dots as Reducing Reagent and Stabilizer
}

\author{
Hoang Vinh Tran*, Anh Duc Chu, Tuan Van Nguyen, Nghia Duc Nguyen, \\ Thu Dieu Le and Chinh Dang Huynh
}

Department of Inorganic Chemistry, School of Chemical Engineering, Hanoi University of Science and Technology (HUST), 1st Dai Co Viet Road, Hanoi, Vietnam

In this work, we have studied effect of various reaction conditions for silver nanoparticles (AgNPs) production by a green and simple method. In our proposed method, silver nanoparticles have been formed and growth under presence of graphene quantum dots (GQDs) as reducing reagent and stabilizer. First, the small sized graphene quantum dots (GQDs) $(5 \mathrm{~nm} \pm 2 \mathrm{~nm})$ with abundant oxygen containing functional groups have been synthesis by hydrothermal method, then GQDs have been used to adsorb Ag ${ }^{+}$ions from solution onto GQDs's surface and after that, $\mathrm{Ag}^{+}$ions have been reduced into silver nanoparticles (AgNPs) by GQDs without adding any reducing reagents. GQDs were coated on the surfaces of the resultant AgNPs, leading to the formation of AgNPs/GQDs nano-hybrids. The optimized reaction conditions (such as pH, reaction time, temperature...) for synthesis of AgNPs using GQDs as a reducing reagent and stabilizer have been investigated. The synthesized AgNPs/GQDs have been characterized by X-ray diffraction (XRD), Transmission Electron Microscopy (TEM), Ultraviolet-visible spectroscopy (UV-Vis), and Dynamic Light Scattering (DLS). Results indicated that mono-dispersed AgNPs were obtained with particles size around 20 nm$40 \mathrm{~nm}$. [doi:10.2320/matertrans.MD201713]

(Received November 30, 2017; Accepted January 24, 2018; Published June 25, 2018)

Keywords: graphene quantum dots (GQDs), silver nanoparticles (AgNPs), green synthesis, nano-hybrids, hydrogen peroxide detection

\section{Introduction}

Recently, metallic nanoparticles have been receiving great attention due to their broad range of usage in different fields such as catalysis, photo-catalysis, chemical and bio-sensing, and nanobiotechnology. Among the low-cost noble metallic nanostructures, silver nanoparticles (Ag-NPs) have been much attended due to their unique properties, such as good electrical conductivity, photoelectrochemical activity, antimicrobial activity, and strong reduction power. The application of AgNPs has been extended to water disinfection in addition to water purification, ${ }^{1)}$ antimicrobial coatings, ${ }^{2,3)}$ wound dressings, ${ }^{4,5)}$ textile industries, ${ }^{6)}$ agriculture, ${ }^{7)}$ sensor ${ }^{8,9)} /$ biosensor $^{10,11)}$ and catalyst. ${ }^{12-14)}$ In addition, AgNPs hold incredible properties that are identified as localized surface Plasmon resonance (LSPR). Due to the sharp LSPR, Ag-NPs can be applied as colorimetric sensors in chemical sensing. ${ }^{15)}$

Silver nanoparticles can be synthesized by chemical, green and microbial approaches. Each approach has its own advantages and limitations. In chemical approach, AgNPs are synthesized by various methods, such as ultrasonic radiation, laser ablation, evaporative cooling, chemical vapor deposition, explosion, impregnation, co-precipitation, solgel, deposition-precipitation, microwave, and microbes. ${ }^{16)}$ In recent years, the green method for synthesis of AgNPs has been attended because of the safety and ecofriendly. Synthesis of AgNPs using plant extracts as the reducing agents and stabilizers is one of the most widely used green methods. ${ }^{17)}$ A variety of plants have been successfully utilized for synthesizing silver metal $\left(\mathrm{Ag}^{0}\right)$ nanoparticles from silver ions $\left(\mathrm{Ag}^{+}\right)$, such as Jatropha curcas, ${ }^{18)}$ Capsicum anuum, ${ }^{16)}$ Argemone mexicana, ${ }^{19)}$ Ocimum sanctum, ${ }^{20)}$ Ficus benghalensis, ${ }^{21)}$ Hibiscus rosa sinensis, ${ }^{22)}$ etc. Recent reports have also shown the potential use of low-cost agricultural

*Corresponding author, E-mail: hoang.tranvinh@hust.edu.vn waste materials. However, the synthesis of AgNPs using using plant extracts as the reducing agents and stabilizers also has some limitations, such as purity of AgNPs product, which limits the application of AgNPs in many industrial fields such as electronics, chemical sensors and biosensors.

Recent time, graphene quantum dots (GQDs) have been known as excellent reducing reagent for synthesis of noble metal nanoparticles. These methods follow green approach but it can improve some disadvantages of using plant extracts, which have been mentioned above. There are several reports on synthesis of GQDs and using its application for synthesis of noble metal nanoparticles such as gold nanoparticles $(\mathrm{AuNPs})^{23)}$ or silver nanoparticles (AgNPs). ${ }^{24-26)}$ However, the influence of reaction factors on the formation of AgNPs still have not presented yet. ${ }^{9,23,25-30)}$ Therefore, the investigation effect of various factors for synthesis of AgNPs using GQDs as reducing reagent and stabilizer is much needed. In this work, we have synthesized of nitrogen graphene quantum dots (GQDs) and using it as reducing reagent and stabilizers for simple and green synthesis of silver nanoparticles (AgNPs/GQDs) by simple hydrothermal process. Various influent factors for synthesis process such as $\mathrm{pH}$, reaction temperature, reaction time and ratio of reaction reagents have been carefully evaluated in the first time was reported.

\section{Experimental Procedure}

\subsection{Materials}

Silver nitrate powder $\left(\mathrm{AgNO}_{3},>99\right.$ mass\%), citric acid $\left(\mathrm{C}_{6} \mathrm{H}_{8} \mathrm{O}_{7} \cdot \mathrm{H}_{2} \mathrm{O}\right)$; urea $\left(\left(\mathrm{NH}_{2}\right)_{2} \mathrm{CO}\right)$; ammonia $\left(\mathrm{NH}_{3}\right)$ solution 28 mass \% and acetic acid $\left(\mathrm{CH}_{3} \mathrm{COOH}\right)$ solution 99 mass\% were purchased from Sigma Aldrich.

\subsection{Synthesis of graphene quantum dots (GQDs)}

$3.44 \mathrm{~g}$ citric acid and $3.005 \mathrm{~g}$ urea were dissolved into $100 \mathrm{ml}$ distillated (D.I) water. The mixture was transferred to 

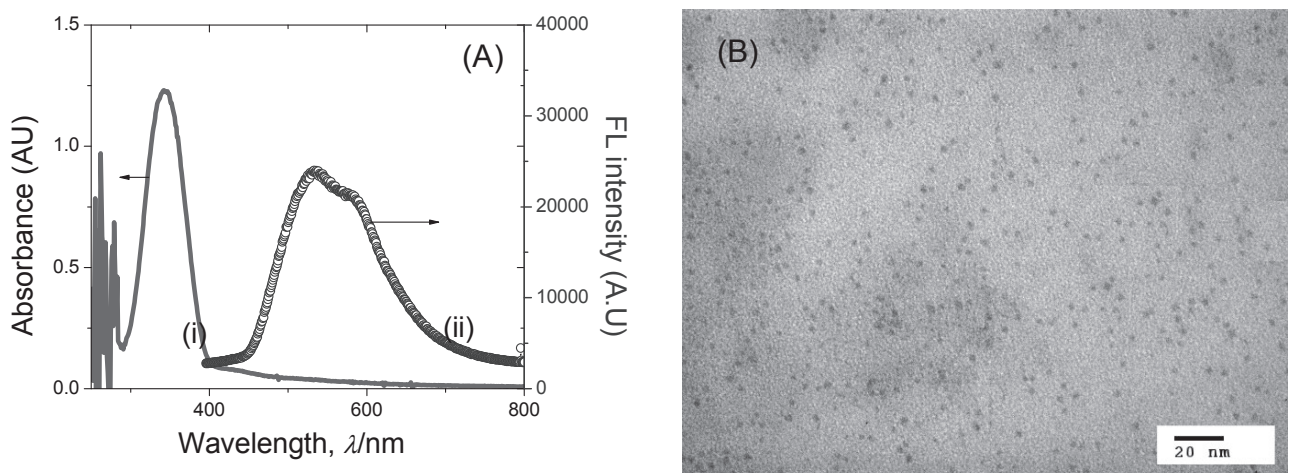

(C)

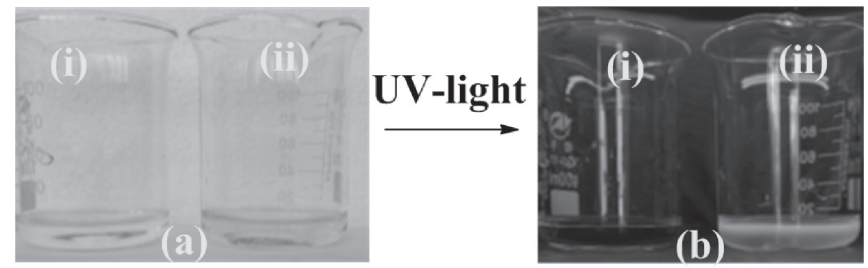

Fig. 1 (A) (i) UV-vis and (ii) FL spectra of GQDs; (B) TEM of GQDs; (C) Digital photographs of (i) water and (ii) GQDs solution under (a) normal light and (b) UV-light.

an autoclave and heated at $160^{\circ} \mathrm{C}$ for $8 \mathrm{~h}$. Then, the mixture was centrifuged at $5000 \mathrm{rpm}$ for $20 \mathrm{~min}$ to remove the big carbon particles. The supernatant containing graphene quantum dots (GQDs) was collected.

\subsection{Synthesis of silver nanoparticles (AgNPs) using GQDs as reducing reagent and stabilizer}

$100 \mu \mathrm{L}$ of GQDs stock solution was added into $3 \mathrm{~mL}$ of D.I water, then $0.1 \mathrm{M} \mathrm{NaOH}$ and $1 \mathrm{M} \mathrm{CH}_{3} \mathrm{COOH}$ solution were used to control $\mathrm{pH}$ of GQDs solution from $\mathrm{pH}=3$ to $\mathrm{pH}=11$. After that, $20 \mu \mathrm{L}$ of $0.1 \mathrm{M} \mathrm{AgNO}_{3}$ solution was added into the GQDs solution. These mixtures were heated at $26^{\circ} \mathrm{C}$ to $90^{\circ} \mathrm{C}$ for 60 minutes to 180 minutes to reduce of $\mathrm{Ag}^{+}$ ion to silver nanoparticles (AgNPs). AgNPs-GQDs solutions then were cooled to room temperature (RT) and stored at $4^{\circ} \mathrm{C}$ for further characterization.

\subsection{Characterization}

Absorbance measurements (UV-Vis) spectra were measured using Agilent $8453 \mathrm{UV}$-Vis spectrophotometer system with the wavelength in a range of 200-1200 nm. Particles size and shape of GQDs, AgNPs/GQDs nanohybrids were analyzed by Transmission Electron Microscope (TEM, JEOL, Voltage: $100 \mathrm{kV}$, magnification: $\times 200,000)$. X-ray Diffraction (XRD) patterns of AgNPs/GQDs was obtained at room temperature by $\mathrm{D} 8$ Advance, Bruker ASX, using $\mathrm{CuK} \alpha$ radiation $(\lambda=1.5406 \AA)$ in the range of $2 \theta=10^{\circ}-60^{\circ}$, and a scanning rate of $0.02 \mathrm{~s}^{-1}$. Particles size distribution was analysed by Dynamic Light Scattering (DLS) on the Nano Partica SZ-100 (HORIBA Scientific, Japan).

\section{Results and Discussions}

\subsection{Characterization of GQDs}

The UV-vis absorption spectrum of GQDs (Fig. 1(A), curve (i)) exhibited two distinct absorption peaks at about $220 \mathrm{~nm}$ and $345 \mathrm{~nm}$, which were attributed to the $\pi-\pi^{*}$ transition of $\mathrm{C}=\mathrm{C}$ and the $\mathrm{n}-\pi^{*}$ transition of $\mathrm{C}=\mathrm{O}$, respectively. The fluorescence emission spectra of the GQDs were recorded and maximum fluorescence emission $(\sim 520 \mathrm{~nm})$ was obtained with an excitation wavelength of $380 \mathrm{~nm}$ (Fig. 1(A), curve (ii)). Figure 1(C) presented the color of water (i) and GQDs solution (ii) under normal light (Fig. 1(C).(a)) and violet light (Fig. 1(C).(b)). It can be seen the emission at blue light of GQDs in solution under excited of the violet light. Moreover, the emission wavelength showed a red shift with increasing excitation wavelength (data not shown). These results are high agreement with previous reports. ${ }^{31-33)}$ According to the TEM image (Fig. 1(B)), the synthesized GQDs were of spherical shape and monodisperse nanoparticles with size distribution in the range of $5 \pm 2 \mathrm{~nm}$.

\subsection{Synthesis of AgNPs using GQDs as reducing reagent and stabilizer \\ 3.2.1 Effect of $\mathrm{pH}$}

Compared with UV-vis spectra of QGDs (Fig. 2(A), curve (a)), it can be seen that the new absorption peak around $\sim 420 \mathrm{~nm}$ to $441 \mathrm{~nm}$ has appeared with mixture of $\mathrm{GQDs}+\mathrm{AgNO}_{3}$ solution after reducing process (Fig. 2(A), curve (b) to curve (f)). These specific peaks can be attributed to AgNPs, which have been formed in presence of GQDs as reducing reagent. However, at low $\mathrm{pH}$, i.e., $\mathrm{pH}=5$ (Fig. 2(A), curve (f)) and $\mathrm{pH}=3$ (Fig. 2(A), curve (g)), the absorption peak of AgNPs was red-shifted due to increasing of AgNPs's size. Effects of pH on the position of AgNPs's plasmon peak (the absorption maximum, $\lambda_{\max }$ ) and peaks's intensity of AgNPs (optical densities at the absorption maximum, OD) were summarized in Fig. 2(B). It is clearly shown that at $\mathrm{pH}=9(\mathrm{pH} 9)$ the position of plasmon peak of AgNPs were lowest wavelength which indicated that the smallest size of AgNPs (Fig. 2(B)) have been formed in solution when the reaction was carried out at this $\mathrm{pH}$. Moreover, plasmon peak intensity of AgNPs at pH9 was also highest, which can be attributed to highest concentration of 

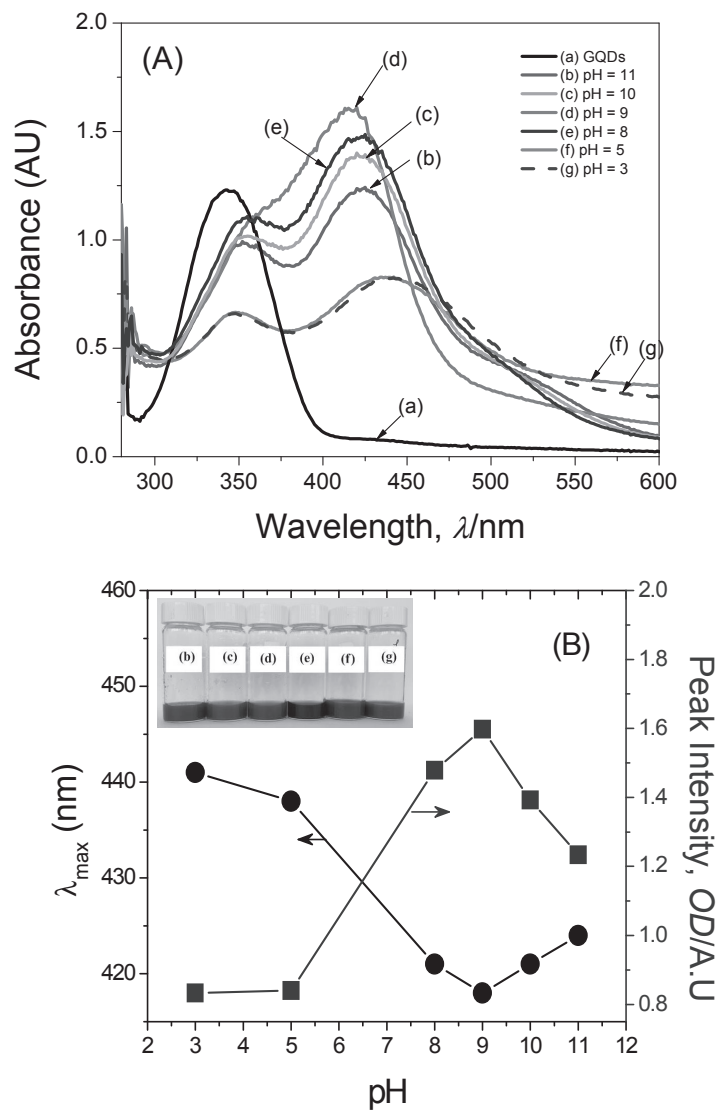

Fig. 2 (A) UV-vis spectra of (a) GQDs solution; curve (b) to (e) AgNPs/ GQDs solutions after reduction at different $\mathrm{pH}$; (B) Effect of $\mathrm{pH}$ on position of AgNPs's plasmon peak (the absorption maximum, $\lambda_{\max }$ ) and peak's intensity (optical density-OD) (insert: digital photographs of the corresponding samples from (A)).

formed AgNPs in solution. These results suggested that $\mathrm{pH} 9$ is suitable medium for the reaction.

\subsubsection{Effect of reaction temperature}

Following Arrhenius equation (eq. (1)), at higher reaction temperature the reducing reaction will be faster:

$$
k=k_{0} \cdot e^{-\frac{E_{\mathrm{a}}}{R \cdot T}}
$$

Where: $k$ is the rate coefficient; $k_{0}$ is a constant, $E_{\mathrm{a}}$ is the activation energy $\left(\mathrm{J} \cdot \mathrm{mol}^{-1}\right), R$ is the universal gas constant $\left(R=8.314 \mathrm{~J} \cdot \mathrm{mol}^{-1} \cdot \mathrm{K}^{-1}\right)$, and $T$ is the temperature (in kelvin, $\mathrm{K})$. In the nanomaterials synthesis reaction, high reaction rate may be leaded faster particle growth rate and giving bigger particle size. However, in this case, GQDs play the role as reducing reagent as well as protectors therefore they inhibited the growth of AgNPs. It can be observed in Fig. 3(A), at low temperature $\left(26^{\circ} \mathrm{C}\right.$ and $\left.40^{\circ} \mathrm{C}\right)$, the optical density of AgNPs was very low indicating that low concentration of formed AgNPs. Figure 3(B) shown that at $90^{\circ} \mathrm{C}$ AgNPs/GQDs sample has lowest absorption peak wavelength $\left(\lambda_{\max } \sim\right.$ $408 \mathrm{~nm}$ ) of AgNPs. Moreover, this sample also has highest peak intensity, with the optical density $\mathrm{OD}_{408 \mathrm{~nm}} \sim 1.6$. These results indicated smallest particles size of formed AgNPs in solution. DLS spectra of AgNPs/GQDs solution (Fig. 3(C)) shown two zones of particle size distributions when reaction temperature were $26^{\circ} \mathrm{C}, 40^{\circ} \mathrm{C}, 60^{\circ} \mathrm{C}$ and $80^{\circ} \mathrm{C}$. At $90^{\circ} \mathrm{C}$ there is only one zone of particles size distribution, which indicated at this temperature, the particles size of AgNPs/GDQs was
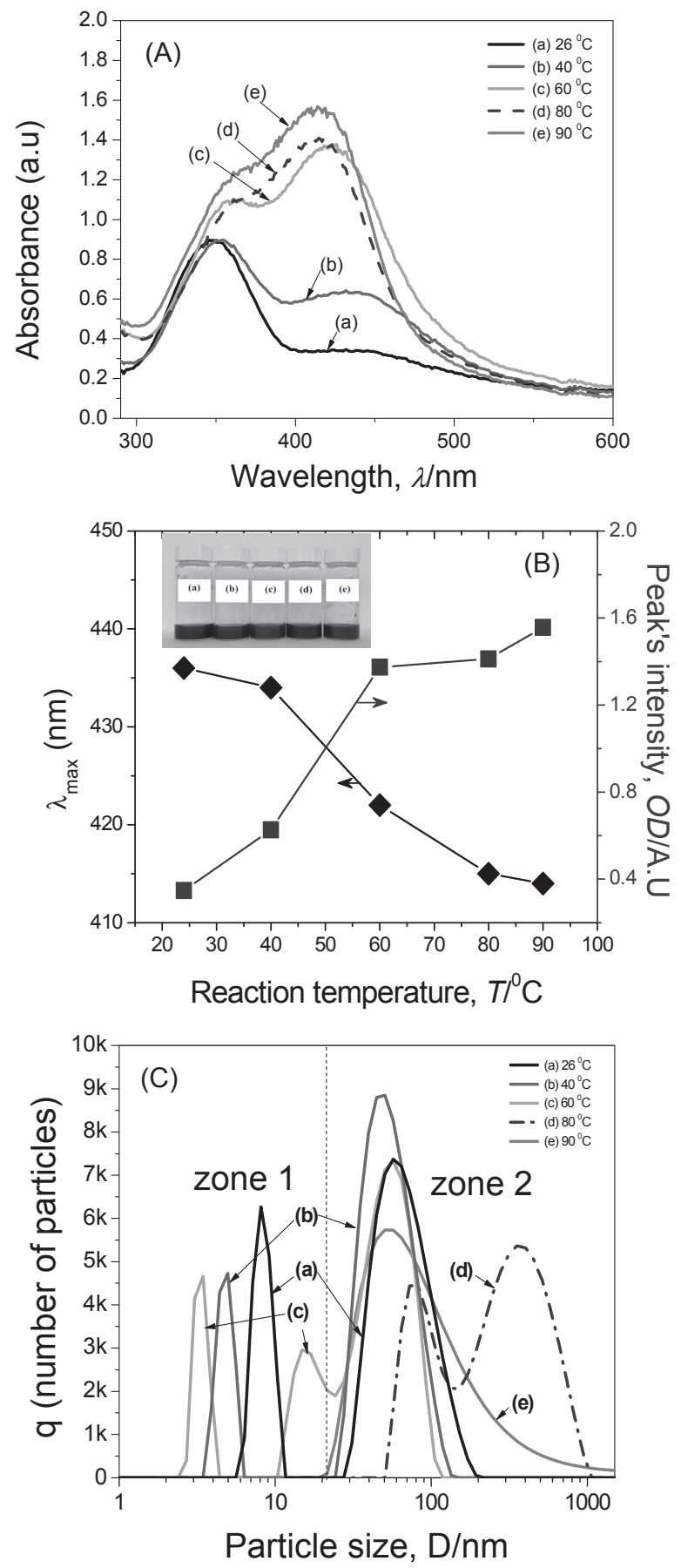

Fig. 3 (A) UV-vis spectra of AgNPs/GQDs solutions were synthesized at different temperature: (a) $26^{\circ} \mathrm{C}$; (b) $40^{\circ} \mathrm{C}$; (c) $60^{\circ} \mathrm{C}$; (d) $80^{\circ} \mathrm{C}$ and (e) $90^{\circ} \mathrm{C}$, respectively; (B) Effect of reaction temperature on position of AgNPs's plasmon peak $\left(\lambda_{\max }\right)$ and peak's intensity (OD) (insert: digital photographs of the corresponding samples from (A)); (C) Particles size distribution of AgNPs/GQDs by DLS method of the corresponding samples from (A).

more homogeneous. Therefore, the temperature of $90^{\circ} \mathrm{C}$ has been used for further experiments.

\subsubsection{Effect of reaction time}

In Fig. 4(A) shown UV-Vis spectra of $\mathrm{AgNO}_{3} / \mathrm{GQDs}$ solution after reducing at $90^{\circ} \mathrm{C}$ for different times. It can be seen AgNPs's plasmon peak $\left(\lambda_{\max }\right)$ and peak's intensity $\left(\mathrm{OD}_{\lambda_{\max }}\right)$ of AgNPs were similar for all samples. However, DLS results (Fig. 4(B)) demonstrated that mean particle sizes were shifted to smaller when reaction time increasing. The 

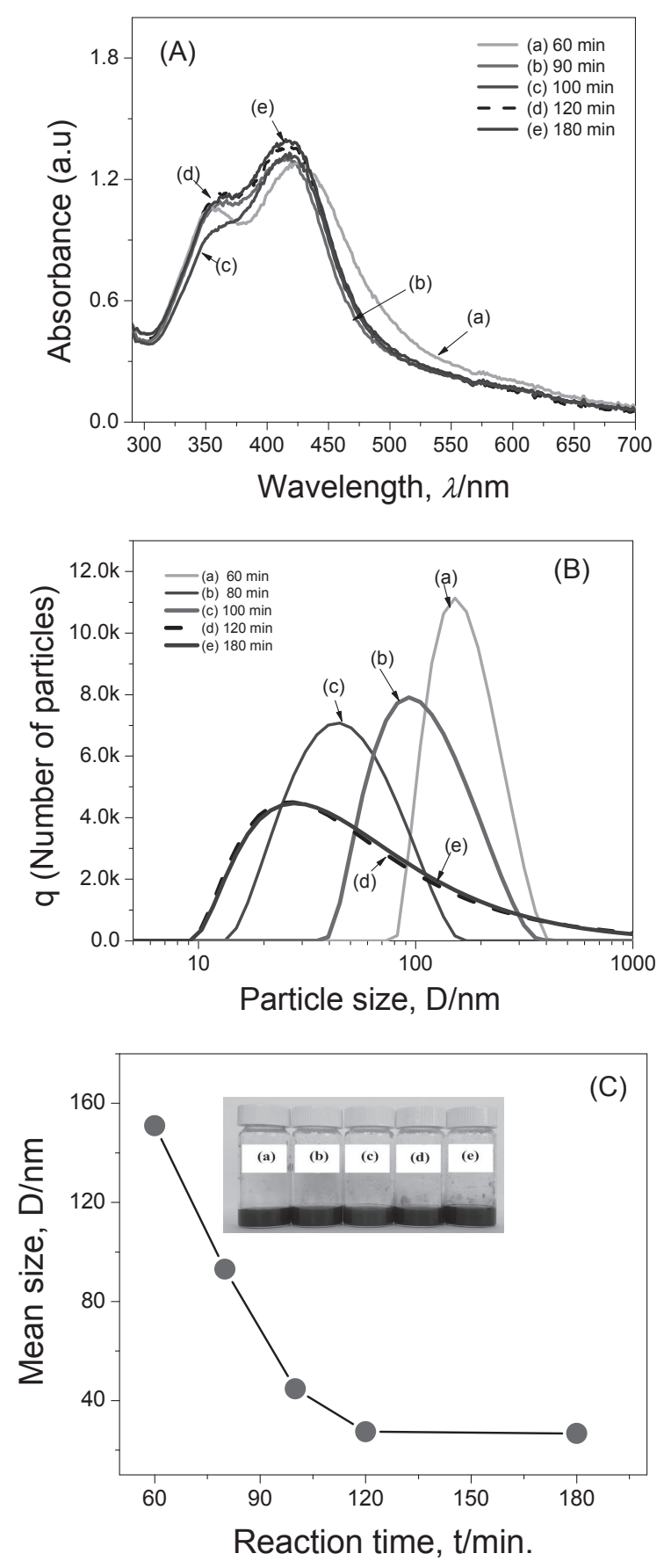

Fig. 4 (A) UV-vis spectra of AgNPs/GQDs solutions were synthesized at $90^{\circ} \mathrm{C}$ for different reaction time: (a) $60 \mathrm{~min}$; (b) $80 \mathrm{~min}$; (c) $100 \mathrm{~min}$; (d) $120 \mathrm{~min}$ and (e) $180 \mathrm{~min}$, respectively; (B) DLS analysis of the corresponding samples from (A); (C) Effect of reaction time on mean particles size of AgNPs/GQDs (insert: digital photographs of the corresponding samples from (A)).

relative of reaction time and mean size of AgNPs/GQDs vs. reaction time were summarized in Fig. 4(C). These results suggested that this reaction needs 2 hours to complete.

\subsubsection{Effect of $\mathrm{Ag}^{+} / \mathrm{GQDs}$ ratio}

In these reactions, $\mathrm{AgNO}_{3}$ (as $\mathrm{Ag}^{+}$ion) plays the role as oxidation reagent and GQDs plays the role as reducing reagent. Therefore, the good product will be obtained if these two reagents are equivalent. However, it is very difficult to estimate the concentration of GQDs in solution as well as estimate the number of reaction centers on one GQD particles. Therefore, the ratio $0.1 \mathrm{M} \mathrm{AgNO}_{3}$ solution volume
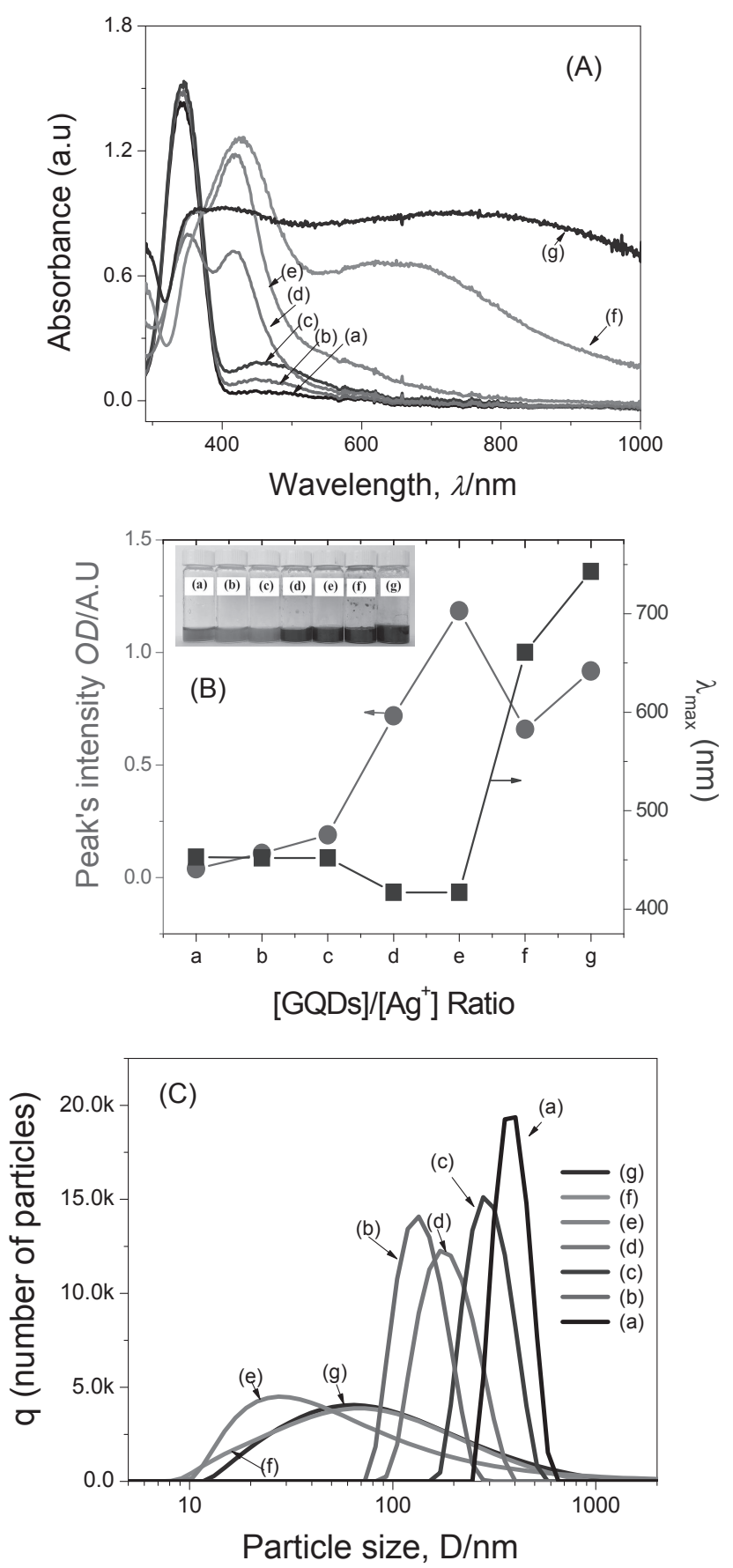

Fig. 5 (A) UV-vis spectra of AgNPs/GQDs solutions were synthesized at $90^{\circ} \mathrm{C}$ for $120 \mathrm{~min}$ with different ratio of $\mathrm{AgNO}_{3} / \mathrm{GQDs}$ solutions. Reaction conditions were described in the text. (B) Effect of volume ratios of $\mathrm{AgNO}_{3}$ and GQDs solutions on position of AgNPs's plasmon peak $\left(\lambda_{\max }\right)$ and peak's intensity (OD) (insert: digital photographs of corresponding samples from (A)); (C) DLS analysis of the corresponding samples from (A), respectively.

and QGDs solution volume were used to find out the optimized point of $\mathrm{AgNO}_{3} / \mathrm{GQD}$ ratio. It can be seen in the UV-Vis spectra (Fig. 5(A)), samples (a) to sample (e), the absorption peaks were around $418 \mathrm{~nm}$ to $425 \mathrm{~nm}$, however, with sample (f) and sample (g) the plasmon peak of AgNPs were shifted to red zone ( $\sim 700 \mathrm{~nm}$ to $\sim 800 \mathrm{~nm})$. These shifts can be attributed to the agglomeration of AgNPs and/or the change of AgNPs's shape. Figure 5(B) shown the relative of $\mathrm{Ag}^{+} /$QGDs ratio on the plasmon peak position of AgNPs $\left(\lambda_{\max }\right)$ and optical densities at the absorption maximum 

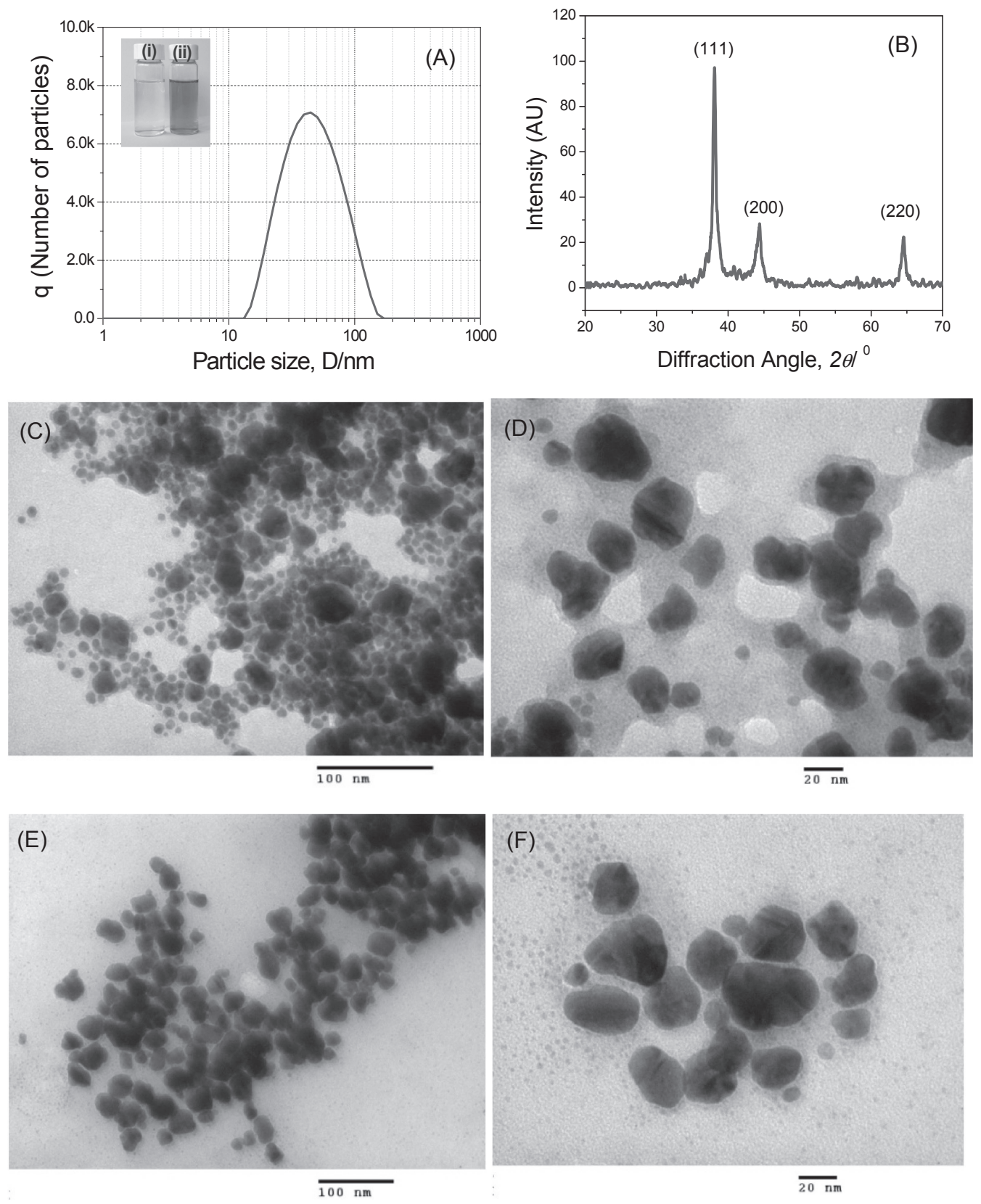

Fig. 6 (A) DLS of AgNPs/GQDs sample was synthesized at optimized conditions (insert: digital photographs of (i) $\mathrm{AgNO}_{3} / \mathrm{GQDs}$ solution before and (ii) after reduction); (B) XRD of AgNPs/GQDs product; (C, D) TEM of AgNPs/GQDs was synthesized at pH 9 and $(\mathrm{E}, \mathrm{F})$ at $\mathrm{pH} 7$ with low magnification $(\mathrm{C}, \mathrm{E})$ and high magnification $(\mathrm{D}, \mathrm{F})$, respectively.

$\left(\mathrm{OD}_{\lambda_{\max }}\right)$. It can be seen that the condition from sample (e) (with $\lambda_{\max }=417 \mathrm{~nm}$ and $\mathrm{OD}_{417 \mathrm{~nm}}$ was 1.185 ) can be used as the optimized condition for this reaction. Size distributions indicated that condition of samples (a), (b), (c) and (d) have given big AgNPs/GQDs's size however size distributions were very narrow (Fig. 5(C)). Condition of sample (g) and sample (f) have very large size distribution (from $10 \mathrm{~nm}$ to $1000 \mathrm{~nm}$ ). Condition of sample (e) has also large size distribution however its mean size around $20 \mathrm{~nm}$. Therefore condition sample (e) was used as optimized $\mathrm{Ag}^{+} / \mathrm{QGDs}$ ratio for synthesis of AgNPs/GQDs.

\subsection{Characterization of AgNPs}

Based on the optimized conditions were obtained above, we have synthesized AgNPs/QGDs for further characterizations. Figure 6(A) shown DLS of AgNPs/GQDs solution with mean particle size of AgNPs/GQDs around $40 \mathrm{~nm}$ was obtained. It can be observed AgNPs/GQDs particles have one distribution zone from $10 \mathrm{~nm}$ to $150 \mathrm{~nm}$. Figure 6(A) (insert) shown $\mathrm{AgNO}_{3} / \mathrm{GQD}$ s solution before as colorless (solution (i)) and after reaction at optimized conditions (solution (ii)) the AgNPs/QGDs was formed as orange color, a specific color of AgNPs colloid. A diffractogram of AgNPs/GQDs is shown on Fig. 6(B), which evidences the typical diffraction planes (111), (200), (220) of the $f_{c c}$ lattice of AgNPs. Line broadening in the pattern can be quantitatively evaluated using Debye-Scherrer equation (eq. (2)), which gives a relationship between peak broadening in XRD and particle size:

$$
D=\frac{k \cdot \lambda}{\beta \cdot \cos \theta}
$$


Where, $D$ is the thickness of the crystal, $k$ is the DebyeScherrer constant $(0.9), \lambda$ is the X-ray wavelength $(0.15406 \mathrm{~nm})$ and $\beta$ is the line broadening in radian obtained from the full width at half maximum, $\theta$ is the Bragg angle. According to Debye-Scherrer equation, particle sizes of AgNPs/GQDs are estimated to be $30 \pm 5 \mathrm{~nm}$. These results are consistent with those obtained by TEM technique (see below). In addition, it can be seen in Fig. 1(C) that no specific XRD peak of GQDs can be seen, possibly this peak is too weak and overlapped by the background signal. The results confirmed the successful preparation of the AgNPs using graphene quantum dots (GQDs) as reducing reagent and stabilizer.

TEM micrographs of AgNPs/GQDs were shown in Fig. 6(C) to Fig. 6(F). At pH 9 (Fig. 6(C) and Fig. 6(D)), it show that the AgNPs have a spherical shape, a smooth surface morphology and particle sizes from $5 \mathrm{~nm}$ to $40 \mathrm{~nm}$. Moreover, it can be observed that almost particles have small size around $10 \mathrm{~nm}$ and a part of particles have size around 20-40 nm, which has high agreement with DLS data above. Figure 6(E) and Fig. 5(F) presented TEM images of AgNPs/GQDs were synthesized at pH7. In Fig. 6(E) (low magnification), it can be observed the homogeneous of AgNPs/QGDs in particles size (around 20-60 nm). However, at high magnification (Fig. 6(F)) we can observe that there are many very small particles of AgNPs/QGDs in the sample. This sample is typical for two distribution zones in DLS results (data not shown). Overall, TEM results indicated the very low aggregation of AgNPs was evidenced, which demonstrates the stabilizing role of GQDs. GQDs plays the role of protectors for AgNPs surface by binding between covalent electron pairs of $-\mathrm{COO}^{-}$and $-\mathrm{NH}_{2}$ group of GQDs with $d$ orbitals of $\mathrm{Ag}^{0}$ atoms and/or $\mathrm{Ag}^{+}$ions on AgNPs's surface and therefore it can prevented the aggregation of AgNPs, so that AgNPs/GQDs can be stored for a long period and be stable in aqueous solutions. . $^{9}, 23,26,27,30$ )

\section{Conclusions}

As summarized, this work has focused on studying the effect of various reaction conditions on the forming of silver nanoparticles (AgNPs) using graphene quantum dots (GQDs) as reducing reagent and stabilizer. The small sized graphene quantum dots (GQDs) with abundant oxygen containing functional groups have been synthesis by hydrothermal method, then GQDs adsorbed $\mathrm{Ag}^{+}$ions and reduced them into silver nanoparticles (AgNPs) without adding any reducing reagents. We have optimized reaction conditions for synthesis of AgNPs using GQDs (such as $\mathrm{pH}$ 9, reaction temperature $90^{\circ} \mathrm{C}$ for 2 hours). The AgNPs/GQDs products with mono-dispersed AgNPs were obtained with particles size around $20 \mathrm{~nm}-40 \mathrm{~nm}$, which has been observed and characterized by TEM, XRD, UV-Vis and DLS analyst.

\section{Acknowledgments}

This research is funded by Vietnam Mistry of Education and Training (MOET) under grant number B2017-BKA-52.

\section{REFERENCES}

1) S.J. Lee, D.N. Heo, M. Heo, M.H. Noh, D. Lee, S.A. Park, J.H. Moon and I.K. Kwon: J. Ind. Eng. Chem. 46 (2017) 273-278.

2) A. Méndez-Albores, S.G.G. Arellano, Y.R. Vidal, J. Torres, Ș. Ţălu, B. Cercado and G. Trejo: J. Alloys Compd. 710 (2017) 302-311.

3) P.A. Abdurazova, U.B. Nazarbek, A.A. Bolysbek, N.K. Sarypbekova, G.S. Kenzhibayeva, G.A. Kambarova, M.S. Sataev, Sh.T. Koshkarbaeva, A.B. Tleuova, S. Perni and P. Prokopovich: Colloids Surf., A 532 (2017) 63-69.

4) R. Singla, S. Soni, P.M. Kulurkar, A. Kumari, S. Mahesh, V. Patial, Y.S. Padwad and S.K. Yadav: Carbohydr. Polym. 155 (2017) 152-162.

5) R.M. Rosa, J.C. Silva, I.S. Sanches and C. Henriques: Mater. Lett. 207 (2017) 145-148.

6) D. Ballottin, S. Fulaz, F. Cabrini, J. Tsukamoto, N. Durán, O.L. Alves and L. Tasic: Mater. Sci. Eng. C 75 (2017) 582-589.

7) Pallavi, C.M. Mehta, R. Srivastava, S. Arora and A.K. Sharma: 3 Biotech 6 (2016) 254.

8) S.N. Wen and J. Shieh: Mater. Trans., JIM 55 (2014) 1800-1805.

9) J. Ge, Y. Li, J. Wang, Y. Pu, W. Xue and X. Liu: J. Alloys Compd. 663 (2016) 166-171.

10) H.V. Tran, C.D. Huynh, H.V. Tran and B. Piro: Arab. J. Chem. (2017) in press. doi:10.1016/j.arabjc.2016.1008.1007.

11) N.D. Nguyen, T.V. Nguyen, A.D. Chu, H.V. Tran, L.T. Tran and C.D. Huynh: Arab. J. Chem. (2018) in press. doi:10.1016/j.arabjc.2017. 1012.1035.

12) E.E. Elemike, D.C. Onwudiwe, A.C. Ekennia, R.C. Ehiri and N.J. Nnaji: Mater. Sci. Eng. C 75 (2017) 980-989.

13) N. Wang, Y. Hu and Z. Zhang: Appl. Clay Sci. 150 (2017) 47-55.

14) M. Ye, R. Wang, Y. Shao, C. Tian, Z. Zheng, X. Gu, W. Wei and A. Wei: J. Photochem. Photobiol., A 351 (2018) 145-153.

15) M.P. Noghabi, M.R. Parizadeh, M.G. Mobarhan, D. Taherzadeh, H.A. Hosseini and M. Darroudi: J. Mol. Struct. 1146 (2017) 499-503.

16) Y. Ping, J. Zhang, T. Xing, G. Chen, R. Tao and K.H. Choo: J. Ind. Eng. Chem. (2017) in press.

17) S. Ahmed, M. Ahmad, B.L. Swami and S. Ikram: J. Adv. Res. 7 (2016) 17-28.

18) H. Bar, D. Kr Bhui, G.P. Sahoo, P. Sarkar, S. Pyne and A. Misra: Colloids Surf., A 348 (2009) 212-216.

19) S. Arokiyaraj, M. Saravanan, N.K. Udaya Prakash, M. Valan Arasu, B. Vijayakumar and S. Vincent: Mater. Res. Bull. 48 (2013) 3323-3327.

20) V. Malapermal, I. Botha, S.B.N. Krishna and J.N. Mbatha: Saudi J. Biol. Sci. 24 (2017) 1294-1305.

21) A. Saxena, R.M. Tripathi, F. Zafar and P. Singh: Mater. Lett. 67 (2012) 91-94.

22) M.R. Bindhu and M. Umadevi: Spectrochim. Acta, Part A 101 (2013) 184-190.

23) Q. Lu, J. Deng, Y. Hou, H. Wang, H. Li, Y. Zhang and S. Yao: Chem. Commun. 51 (2015) 7164-7167.

24) S. Chen, X. Hai, X.W. Chen and J.H. Wang: Anal. Chem. 86 (2014) 6689-6694.

25) S. Chen, Y. Quan, Y.L. Yu and J.H. Wang: ACS Biomater. Sci. Eng. 3 (2017) 313-321.

26) Y. Su, B. Shi, S. Liao, J. Zhao, L. Chen and S. Zhao: ACS Biomater. Sci. Eng. 4 (2016) 1728-1735.

27) J.C. Jin, Z.Q. Xu, H.F. Zou, Z.Q. Zhou, Q.Q. Yang, B.B. Wang, F.L. Jiang and Y. Liu: RSC Advances 6 (2016) 76989-76995.

28) L. Yang, X. Liu, Q. Lu, N. Huang, M. Liu, Y. Zhang and S. Yao: Anal. Chim. Acta 930 (2016) 23-30.

29) S. Zhang, X. Liu, N. Huang, Q. Lu, M. Liu, H. Li, Y. Zhang and S. Yao: Electrochim. Acta 211 (2016) 36-43.

30) T. Liu, J.X. Dong, S.G. Liu, N. Li, S.M. Lin, Y.Z. Fan, J.L. Lei, H.Q. Luo and N.B. Li: J. Hazard. Mater. 322 (2017) 430-436.

31) H. Li, X. He, Z. Kang, H. Huang, Y. Liu, J. Liu, S. Lian, C.H.A. Tsang, X. Yang and S.T. Lee: Angew. Chem. Int. Ed. 49 (2010) 4430-4434.

32) M.J. Krysmann, A. Kelarakis, P. Dallas and E.P. Giannelis: J. Am. Chem. Soc. 134 (2012) 747-750.

33) Z. Lin, W. Xue, H. Chen and J.M. Lin: Chem. Commun. 48 (2012) 1051-1053. 